\title{
Challenging behaviour and learning disabilities: summary of NICE guidance
}

\author{
Stephen Pilling director, professor ${ }^{12}$, Elena Marcus research assistant ${ }^{12}$, Craig Whittington associate \\ director, senior research associate ${ }^{12}$, Glynis Murphy co-director, professor ${ }^{3}$, On behalf of the \\ Guideline Development Group
}

${ }^{1}$ National Collaborating Centre for Mental Health, University College London, London WC1E 7HB, UK; ${ }^{2}$ Centre for Outcomes Research and Effectiveness, University College London; ${ }^{3}$ Tizard Centre, University of Kent, Canterbury, UK

This is one of a series of $B M J$ summaries of new guidelines based on the best available evidence; they highlight important recommendations for clinical practice, especially where uncertainty or controversy exists.

It is relatively common for people with a learning disability to develop behaviour that challenges. In educational, health, and social care settings, prevalence rates of challenging behaviour in people with learning disabilities vary from $10 \%$ to $15 \%,{ }^{12}$ with rates peaking between the ages of 20 and 49 years. Rates are higher in people with more severe disabilities and within inpatient settings. ${ }^{1}$ Behaviour that challenges includes aggression, self injury, stereotypic behaviour, and withdrawal, which often result from the interaction of personal and environmental factors. Such behaviour can have detrimental consequences for the person, including lower quality of life, restrictive practices, physical abuse, and out of area placements. ${ }^{1}$ This article summarises the most recent recommendations from the National Institute for Health and Care Excellence (NICE) on challenging behaviour and learning disabilities in adults, children, and young people. ${ }^{3}$

\section{Recommendations}

NICE recommendations are based on systematic reviews of the best available evidence and explicit consideration of cost effectiveness. When minimal evidence is available, recommendations are based on the Guideline Development Group's experience and opinion of what constitutes good practice. Evidence levels for the recommendations are given in italic in square brackets.

\section{General principles of care}

Working with people with a learning disability and behaviour that challenges, and with their families and carers

- When providing support and interventions:

-Take into account the severity of people's learning disability and their developmental stage, any communication difficulties, and physical or mental health problems

-Aim to provide support and interventions in the least restrictive setting (such as the person's home or in other places where the person regularly spends time)

-Aim to prevent, reduce, or stop the development of future episodes of behaviour that challenges

-Offer support and interventions respectfully and aim to improve quality of life

-Ensure that the focus is on improving people's support and increasing their skills rather than changing people

-Ensure that people know whom to contact if they are worried about care or interventions, including their right to a second opinion

-Offer independent advocacy to the person and to family members or carers. [Based on two existing systematic reviews, ${ }^{45}$ patient expert advisory groups, and the experience and opinion of the Guideline Development $\operatorname{Group}(G D G)$.]

\section{Team working}

- If initial assessment and management have not been effective, or the person has more complex needs, health 


\section{The bottom line}

- Include the person with learning disabilities and his or her family and carers in assessments and interventions

- Undertake functional assessments and implement behaviour support plans linked to these

- Provide the least restrictive behavioural, cognitive behavioural, and psychosocial interventions, as close to home as possible

- Reserve drugs as a treatment option for severe aggression or self injury, and only in combination with a behavioural, cognitive behavioural, or psychosocial intervention

- Aim to increase quality of life as well as reducing behaviour that challenges

\section{How patients were involved in the creation of this article}

Three lay committee members with specific knowledge and experience of challenging behaviour in people with a learning disability contributed to the formulation of the recommendations summarised in this article. People with learning disabilities and carers of people with learning disabilities and behaviour that challenges took part in focus groups that informed the development of recommendations summarised in this article.

and social care provider organisations should ensure that teams providing care have prompt and coordinated access to specialist assessment, support, and intervention services. These services should provide advice, supervision, and training from a range of staff to support any care or intervention, including psychologists, psychiatrists, behavioural analysts, nurses, social care staff, speech and language therapists, educational staff, occupational therapists, physicians, paediatricians, and pharmacists. [Based on the experience and opinion of the GDG.]

\section{Staff training, supervision, and support}

- Health and social care provider organisations should ensure that all staff working with people with a learning disability and behaviour that challenges are trained to deliver proactive strategies to reduce the risk of this behaviour, including:

-Developing personalised daily activities

-Adapting a person's environment and routine

-Helping the person develop an alternative behaviour to achieve the same purpose by developing a new skill (for example, improved communication or emotional regulation)

-Involving people and their family members or carers in planning support and interventions

-Using strategies designed to calm and divert people who show early signs of distress

-Delivering reactive strategies. [Based on an existing systematic review of non-randomised studies ${ }^{6}$ and the experience and opinion of the GDG.]

\section{Support and interventions for family members or carers}

- When providing support to family members or carers (including siblings):

-Recognise the impact of living with or caring for a person with a learning disability and behaviour that challenges

-Explain how to access family advocacy

-Consider family support and information groups if there is a risk of behaviour that challenges or if it is emerging

-Consider formal support through disability specific support groups for family members or carers and regular assessment of the extent and severity of the behaviour that challenges
-Provide skills training and emotional support to help family and carers take part in and support interventions. [Based on very low to moderate quality evidence from randomised controlled trials (RCTS) and the experience and opinion of the GDG.]

\section{Early identification of the emergence of initial behaviour that challenges}

- Everyone involved in caring for and supporting people with a learning disability (including family members and carers) should understand the risk of behaviour that challenges and that it often develops gradually. Pay attention to and record factors that may increase this risk, including:

-Personal factors, such as:

A severe learning disability

Autism

Dementia

Communication difficulties (expressive and receptive)

Visual impairment (which may lead to increased self injury and stereotypy)

Physical health problems

Variations with age (peaking in the teens and 20s).

-Environmental factors, such as:

Abusive or restrictive social environments

Environments with too little or too much sensory stimulation and those with low engagement levels (for example, little interaction with staff)

Developmentally inappropriate environments (for example, a curriculum that makes too many demands on a child or young person)

Environments where disrespectful social relationships and poor communication are typical, or where staff do not have the capacity or resources to respond to people's needs

Changes to the person's environment (for example, major staff changes or a move to a new care setting).

[Based on very low to moderate quality evidence from observational studies.]

\section{Physical healthcare}

- GPs should offer an annual physical health check to children, young people, and adults with a learning disability in all settings, using a standardised template (such as the 
Cardiff health check template). ${ }^{7}$ Undertake this with a family member, carer, healthcare professional, or social care practitioner who knows the person and include: -A review of any known or emerging behaviour that challenges and how it may be linked to any physical health problems (such as hearing impairment or pain-for example, toothache)

-A physical health review

-A review of all current health interventions, including drugs and related side effects, adverse events, and drug interactions and adherence

-An agreed and shared care plan for managing any physical health problems (including pain). [Based on very low to moderate quality evidence from RCTs and the experience and opinion of the GDG.]

\section{Assessment}

\section{The assessment process}

- When assessing behaviour that challenges, ensure that: -The person being assessed remains at the centre of concern and is supported throughout the process, and that family members and carers are fully involved

-All personal and environmental factors (past and current) that may lead to behaviour that challenges are taken into account

-Assessment is a flexible process, because factors that trigger and maintain behaviour may change over time

-Assessments are reviewed after any serious change in behaviour

-Assessments focus on the outcomes of reducing behaviour that challenges and improving quality of life

-The resilience, resources, and skills of family members and carers are taken into account

-The capacity, sustainability, and commitment of the staff delivering the behaviour support plan are taken into account. [Based on the experience and opinion of the $G D G$.]

\section{Risk assessment}

- Assess and regularly review the following areas of risks during any assessment of behaviour that challenges: -Suicidal ideation, self harm (particularly in people with depression), and self injury

-Harm to others

-Self neglect

-Breakdown of family or residential support

-Exploitation, abuse, or neglect by others

-Rapid escalation of the behaviour that challenges.

Ensure that the behaviour support plan includes risk management. [Based on the experience and opinion of the GDG.]

\section{Functional assessment of behaviour}

- Vary the complexity and intensity of the functional assessment according to the complexity and intensity of the behaviour that challenges, following a phased approach as set out below:
-Gather pre-assessment data to help shape the focus and level of the assessment

-For recent onset behaviour that challenges, consider brief structured assessments such as the Functional Analysis Screening Tool ${ }^{8}$ or Motivation Assessment Scale ${ }^{9}$ to identify associations between the behaviour and what triggers and reinforces it

-Take into account whether any important changes to the person's environment and physical or psychological health are associated with the development or maintenance of recent onset behaviour that challenges or marked changes in patterns of existing behaviours

-Consider in-depth assessment involving interviews with family members, carers, and others; direct observations; structured record keeping; questionnaires; and reviews of case records

-If a mental health problem might underlie behaviour that challenges, consider initial screening using assessment scales such as the Diagnostic Assessment Schedule for the Severely Handicapped-II, ${ }^{10}$ Psychiatric Assessment Schedule for Adults with a Developmental Disability, ${ }^{11}$ or the Psychopathology Instrument for Mentally Retarded Adults $^{12}$ and seek expert opinion. [Based on low to moderate quality psychometric evidence and the experience and opinion of the GDG.]

\section{Psychological and environmental interventions}

\section{Early intervention for children and their parents or carers}

- Consider parent training programmes for parents or carers of children with a learning disability who are aged under 12 years with emerging, or at risk of developing, behaviour that challenges. [Based on very low to moderate quality evidence from RCTs.]

\section{Interventions for behaviour that challenges}

- Consider personalised interventions that are based on behavioural and cognitive behavioural principles and a functional assessment of behaviour, are tailored to the range of settings in which they spend time, and consist of: -Clear targeted behaviours with agreed outcomes

-Assessment and modification of environmental factors that could trigger or maintain the behaviour (for example, altering task demands for avoidant behaviours)

-Addressing staff and family member or carer responses to behaviour that challenges

-A clear schedule of reinforcement of desired behaviour and the capacity to offer reinforcement promptly

-A specified timescale to meet intervention goals (modifying intervention strategies that do not lead to change within a specified time). [Based on very low to low quality evidence from RCTs and the experience and opinion of the $G D G$.]

\section{Drugs}

- Consider antipsychotic drugs to manage behaviour that challenges, only in combination with psychological or other interventions, and only if: 
-Psychological or other interventions alone do not produce change within an agreed time, or

-Treatment for any coexisting mental or physical health problem has not led to a reduction in the behaviour, or

-The risk to the person or others is severe (for example, because of aggression or self injury). [Based on very low to low quality evidence from RCTs and the experience and opinion of the GDG.]

\section{Overcoming barriers}

There are three major barriers to effective implementation of this guideline. The first is the currently inadequate structure and resourcing of services, including the specialist community learning disability team. The capacity to deliver services is also severely hindered by the lack of appropriate support close to people's homes. Limitations in staff competency and high quality training present a third major barrier. Lack of training can lead to poor recognition of the behaviour that challenges and the underlying causes of that behaviour, as well as limited skills and knowledge in implementing appropriate treatment plans. Failure in these areas leads to over-reliance on drugs, which for many people will have limited effectiveness and may lead to serious side effects. ${ }^{1314}$

The members of the Guideline Development Group were Glynis Murphy (chair, professor of clinical psychology and disability), Steve Pilling (facilitator, professor of clinical psychology and clinical effectiveness), David Allen (professor in the clinical psychology of intellectual disability), Katherine Andrea (project manager), David Branford (pharmacist), Alick Bush (clinical psychologist), Carole Buckley (general practitioner), Vivien Cooper (lay member and CEO of The Challenging Behaviour Foundation), Parmi Dhennsa (lay member), Jo Dwyer (occupational therapist), David Glynn (health economist), Bronwyn Harrison (systematic reviewer), Angela Hassiotis (psychiatrist), Phil Howell (manager, Physical Interventions Accreditation Scheme), Simon Jones (head of behavioural support, Care UK), Elena Marcus (research assistant), Ifigeneia Mavranezouli (health economist), Richard Mills (research director, Research Autism), David Newton (independent adult safeguarding coordinator), Steve Noone (clinical psychologist), Cheryl Palmer (research assistant), Phil Perkins (nurse), Victoria Slonims (speech and language therapist), Sarah Stockton (information scientist), Clare Taylor (editor), Craig Whittington (systematic reviewer), and Keith Wyncoll (lay member).

Contributors: All authors were part of the Guideline Development Group that made the recommendations described in this summary. EM and SP drafted the summary and CW and GM revised it critically for important intellectual content. All authors read the final draft of the manuscript and agreed for it to be published. All authors agree to be accountable for all aspects of the work in ensuring that questions related to the accuracy or integrity of any part of the work are appropriately investigated and resolved. All authors are guarantors.
Funding: SP, CW, and EM are employees of University College London, funded by the National Institute for Health and Care Excellence (NICE), which is commissioned and funded by the Department of Health to develop clinical guidelines. GM received a fee from NICE for chairing the Guideline Development Group that made the recommendations described in this summary. No authors received specific funding to write this summary.

Competing interests: We declare the following interests based on National Institute for Health and Care Excellence's policy on conflicts of interests (www.nice.org.uk/Media/Default/About/Who-we-are/Policiesand-procedures/code-of-practice-for-declaring-and-managing-conflictsof-interest.pdf). SP, CW, and EM had support from the National Collaborating Centre for Mental Health, which was in receipt of funding from NICE, for the submitted work; there are no other relationships or activities that could appear to have influenced the submitted work. The authors' full statements can be viewed at: www.bmj.com/content/bmj/ 350/bmj.h2652/related\#datasupp.

Provenance and peer review: Commissioned; not externally peer reviewed.

Emerson E, Einfeld S. Challenging behaviour. 3rd ed. Cambridge University Press, 2011 2 Lowe K, Allen D, Jones E, et al. Challenging behaviours: prevalence and topographies. $J$ Intellect Disabil Res 2007;51:625-36.

3 National Institute for Health and Care Excellence. Challenging behaviour and learning disabilities: prevention and interventions for people with learning disabilities whose behaviour challenges. (NICE guideline 11). 2015. www.nice.org.uk/guidance/ng11.

4 Griffith GM, Hutchinson L, Hastings RP. "I'm not a patient, I'm a person:" the experiences of individuals with intellectual disabilities and challenging behavior-a thematic synthesis of qualitative studies. Clin Psychol 2013;20:469-88.

5 Griffith G, Hastings R. "He's hard work, but he's worth it." The experience of caregivers of individuals with intellectual disabilities and challenging behaviour: a meta-synthesis of qualitative research. J Appl Res Intellect Disabil 2013;27:401-19.

6 MacDonald A, McGill P. Outcomes of staff training in positive behaviour support: a systematic review. J Dev Phys Disabil 2013;25:17-33.

7 Cardiff health check protocol. Royal College of General Practitioners. 2010. www.rcgp. org.uk/ /media/Files/CIRC/CIRC-76-80/CIRCA\%20StepbyStepGuideforPracticesOctober\% 2010.ashx.

8 Iwata BA, Deleon IG, Roscoe EM. Reliability and validity of the functional analysis screening tool. J Appl Behavior Anal 2013;46:271-84.

9 Durand VM, Crimmins DB. Motivation assessment scale. Monaco and Associates; 1992

10 Bamburg JW, Cherry KE, Matson JL, et al. Assessment of schizophrenia in persons with severe and profound mental retardation using the Diagnostic Assessment for the Severely Handicapped-II (DASH-II). J Dev Phys Disabil 2001;13:319-31.

11 Moss S, Prosser H, Costello H, et al. Reliability and validity of the PAS-ADD checklist for detecting psychiatric disorders in adults with intellectual disability. $J$ Intellect Disabil Res 1998;42:173-83.

12 Linaker OM, Helle J. Validity of the schizophrenia diagnosis of the Psychopathology Instrument for Mentally Retarded Adults (PIMRA): a comparison of schizophrenic patients with and without mental retardation. Res Dev Disabil 1994;15:473-86.

13 Brylewski J, Duggan L. Antipsychotic medication for challenging behaviour in people with learning disability. Cochrane Database Syst Rev 2004;3:CD000377.

14 McIntyre RS, Mancini DA, Basile VS. Mechanisms of antipsychotic-induced weight gain. $J$ Clin Psychiatry 2001;62:23-9.

15 Department of Health. Transforming care: a national response to Winterbourne View hospital. 2012. www.gov.uk/government/uploads/system/uploads/attachment_data/file/ 213215/final-report.pdf.

16 Sanders MR, Kirby JN, Tellegen CL, et al. The triple P-positive parenting program: a systematic review and meta-analysis of a multi-level system of parenting support. Clin Psychol Rev 2014;34:337-57.

17 Webster-Stratton $C$. The Incredible Years parents, teachers and children's training series: program content, methods, research and dissemination 1980-2011. Incredible Years, 2012.

18 GRADE Working Group. 2015. www.gradeworkinggroup.org/index.htm.

Cite this as: BMJ 2015;350:h2652

(c) BMJ Publishing Group Ltd 2015 


\section{Further information on the guidance}

The management of behaviour that challenges in health and social care organisations is currently a matter of national concern. The Winterbourne View abuse scandal, ${ }^{15}$ where people with learning disabilities in a private hospital were systematically physically and psychologically abused over a considerable period of time, provided an example of extraordinarily negligent care. Although concerns were raised with regulatory bodies, the problem did not come to public attention until an undercover TV investigation was carried out. Overall this event has highlighted serious inadequacies in the capacity of services to prevent or effectively respond to behaviours that challenge. This guideline sets out a clear approach to assessing behaviour that challenges, with an emphasis on functional analysis. Training for parents of younger children with a learning disability has emerged as a new intervention that may have a major role in preventing the development of behaviour that challenges. ${ }^{1617}$ The guideline also stresses the importance of annual physical health checks in primary care, and that inadequately treated physical health problems often underlie behaviour that challenges.

\section{Methods}

This guideline was developed by the National Collaborating Centre for Mental Health according to the methods specified in the National Institute for Health and Care Excellence (NICE) guideline manual 2012 (www.nice.org.uk/guidelinesmanual). The guideline review process involved comprehensive and systematic literature searches to identify relevant evidence for the clinical and economic reviews. The limited evidence base required the development and refinement of existing methods, including procedures for extrapolating from other datasets and the incorporation and adaptation of recommendations from other NICE guidelines. For one review question, a formal consensus method based on the modified nominal group technique was also adopted. The quality of the clinical evidence was appraised using methods specified by the GRADE working group. ${ }^{18}$

The Guideline Development Group (GDG) comprised a multidisciplinary team of healthcare professionals from psychology, psychiatry, pharmacology, occupational therapy, general practice, nursing, commissioning, social care, and speech and language therapy, as well as carer representatives; all had specific knowledge or experience of behaviour that challenges in people with a learning disability. The GDG reviewed the evidence and developed subsequent recommendations. The guideline then went through an external consultation with stakeholders. The GDG considered the stakeholders' comments, reanalysed data where necessary, and modified the guideline as appropriate. NICE has produced four different versions of the guideline: a full version; a short version; a version for the public (www.nice.org.uk/guidance/ $\mathrm{NG11/InformationForPublic);} \mathrm{and} \mathrm{an} \mathrm{easy} \mathrm{to} \mathrm{read} \mathrm{version} \mathrm{for} \mathrm{children,} \mathrm{young} \mathrm{people,} \mathrm{and} \mathrm{adults} \mathrm{with} \mathrm{challenging} \mathrm{behaviour} \mathrm{and} \mathrm{a} \mathrm{learning}$ disability (www.nice.org.uk/guidance/NG11/EasyRead). All these versions, as well as a pathway, are available from the NICE website. Further updates of the guideline will be produced as part of NICE's guideline development programme.

\section{Future research}

- Can positive behaviour support provided to children under 5 years with a learning disability reduce the risk of developing behaviour that challenges?

- For adults with a learning disability, are interventions based on the science and practice of applied behavioural analysis or antipsychotic drugs, or a combination of these, effective in reducing the frequency and severity of behaviour that challenges?

- Does providing care where people live, compared with out of area placement, improve both the clinical and cost effectiveness of care?

- What factors (including service organisation and management, staff composition, training and supervision, and the content of care and support) are associated with sustained high quality residential care? 